\title{
Together we stand, apart we fall: how cell-to-cell contact/interplay provides resistance to ferroptosis
}

\author{
Milica Vucetic ${ }^{1}$, Boutaina Daher ${ }^{1}$, Shamir Cassim¹, Willian Meira ${ }^{1}$ and Jacques Pouyssegur (i) ${ }^{1,2}$
}

\begin{abstract}
Contextualisation of the new type of cell death called "ferroptosis" opened a completely new avenue for the development of anti-cancer therapies. Cumulative fundamental research dating back to the mid-20th century, crowned by the extraordinary work of the group led by Dr. Stockwell from Columbia University in 2012, finally got its candidature to be applied in the clinical settings. Although the potential for clinical importance is undoubtedly growing every day, as showed by the increasing number of papers dealing with ferroptosis and its applications, long experience of cancer research and treatment taught us that caution is still necessary. The plasticity of the tumour cells, particularly acute, along with its involvement in the resistance mechanisms, that have been seen, to greater or lesser extent, for almost all currently used therapies, represents the biggest fascinations in biomedical research field and also the biggest challenge to achieving cures in cancer patients. Accordingly, the main features of fundamental research have to be vigilance and anticipation. In this review, we tried to summarize the literature data, accumulated in the past couple of years, which point out the pitfalls in which "ferroptosis inducers" can fall if used prematurely in the clinical settings, but at the same time can provide a great advantage in the exhausting battle with cancer resistance. This is the first comprehensive review focusing on the effects of the cell-to-cell contact/interplay in the development of resistance to ferroptosis, while the contribution of cell-born factors has been summarized previously so here we just listed them.
\end{abstract}

\section{Facts}

- Newly contextualized type of cell death termed "ferroptosis", although highly promising as a strategy for eradication of the tumour cells, still has not been fully understood in the in vivo conditions.

- Cell-to-cell contact influences the sensitivity of the cancer cells to ferroptosis

- Cancer cell resistance to ferroptosis at higher density has been ascribed to the activation of the Hippo pathway and its intersections with other signalling pathways/ processes such as mTORC1, EMT, etc.

- The Hippo pathway has been described to contribute

Correspondence: Milica Vucetic (milica@centrescientifique.mc) or

Jacques Pouyssegur (jacques.pouyssegur@unice.fr)

'Medical Biology Department, Centre Scientifique de Monaco (CSM), Monaco, Monaco

University Côte d'Azur, Institute for Research on Cancer \& Aging (IRCAN), CNRS, INSERM, Centre A. Lacassagne, Nice, France

Edited by U. Maurer to cell density effects, although this seemingly just applies to adherent cancer cells, and as such might be cell context dependent.

- Besides direct contact, metabolic shuttling (cell-tocell interplay) between the tumour and stromal cells fundamentally changes the response of these cells to ferroptosis induction.

\section{Open questions}

- Is Hippo pathway the only/main mechanism involved in the high-density-induced resistance to ferroptosis of the adherent cells?

- Does the cell-to-cell contact of the non-adherent cells have the same effect on the ferroptosis sensitivity?

- How the cell-to-cell interplay can be used to enhance the efficacy of the ferroptosis inducers, other chemotherapeutics and/or immunotherapy? 


\section{Introduction}

The (patho)physiology of the cancer development and progression represents an extremely complex field, which prevents us, until this date, to form comprehensive definition of the disease. Surprisingly, in the review from 2002, Green and Evan ${ }^{1}$ hypothesized that the main driving force for all cancer types is "unique, very rare, simultaneous acquisition of the two cooperating conditions-deregulated cell proliferation and suppressed apoptosis". According to the authors, all the other driving forces, seen on this "established cancer platform", which consequently lead to divergences from the common evolutionary trajectory, are the result of the changing selective pressure in the expanding cell population. Although the difference between this two-hallmark and, widely accepted, six(ten)-hallmark ${ }^{2,3}$ description of cancer cell can be a matter of debate, when it comes to anti-cancer treatment, only one question is really important-how to specifically kill cancer cells?

Early studies dealing with the involvement of apoptosis in the neoplastic transformation and cancer progression ${ }^{4}$, together with cloning and characterization of the first oncogenes (such as $b c l-2$ ) and tumour suppressor genes $(\text { such as } p 53)^{5}$, sparked optimism in the cancer research community. Thus, the focus was placed on the understanding and exploiting strategies for (re)induction of the cell death program in cancers, and eventually, the development of new and more effective anti-cancer regiments $^{6-9}$. In parallel, scientists were learning more about the complexity of the apoptosis, different intrinsic/ extrinsic signals that can trigger this type of cell death, and unfortunately, the resistance mechanisms through the incredible power of the Darwinian selection.

Similar optimism, when it comes to anti-cancer therapeutics, appeared in the scientific community once again quite recently, when the group of Dr. Brent Stockwell at Columbia University contextualized a new type of cell death, called "ferroptosis" ${ }^{10}$. According to a growing number of data, initial optimism seems to be justified since ferroptosis-induction proved to be a highly promising strategy for eradication of the cancer cells. However, in order to avoid the trap of oversimplification, special attention has to be placed on the multiple potential mechanisms that confer resistance to this type of cell death.

The aim of this review is to summarize the recent findings suggesting that cell-to-cell contact and interplay impact tumour cell sensitivity to ferroptosis. Furthermore, we tried to place these effects in the context of cellular features that might be perceived as (only) cell-born treats, and to point out the most important avenues that remain to be examined.

\section{Ferroptosis-in the perspective}

Although the contextualization of ferroptosis, as a new type of cell death happened just a couple years ago ${ }^{10}$, the research leading to this extraordinary discovery dates back several decades in the past and is marked by a couple of major milestones that paved the way to the ferroptosis concept as we know it today ${ }^{11}$. The first major discovery happened in the mid-20th century by Dr. Harry Eagle who, by investigating the nutritional requirements for survival and growth of mammalian cells in in vitro conditions, showed that the classified non-essential amino acid-cysteine $(\mathrm{CySH})$, is not only essential for proliferation of cells in culture, but its absence inevitably leads to very specific type of cell death ${ }^{12-14}$. Two decades later, exceptional work of the Japanese group led by Dr Shiro Bannai, depicted the essential connection between $\mathrm{CySH}-$ starvation, depletion of intracellular glutathione (GSH) and consequent accumulation of reactive oxygen species (ROS), and ultimately, cell death ${ }^{15}$. Moreover, this study added up to the general knowledge of lipid-based oxidative damage of the cell that had been started independently by the groups of Dr. Peterson and Dr. Rothberg ${ }^{16,17}$ and continued until $80 \mathrm{~s}$, when the process of lipid peroxidation was officially recognized as a major form of oxidative damage of lipid compartments within the cell, correlating with many different pathologies ${ }^{18-20}$. Last milestone in the ferroptosis research, which happened about the same time, is the seminal discovery, isolation and purification of phospholipid hydroperoxide glutathione peroxidase 4 (PHGPX4 aka GPx4) by Dr. Fulvio Ursini and coworkers ${ }^{21}$. Importantly, the loss/inhibition of this enzyme leading to specific type of non-apoptotic cell death was actually the first step toward ferroptosis contextualization $^{22,23}$. These four major milestones that happened over the 30-year long period had massive impact on our understanding of oxidative damage and its involvement in the process of cell death; however it took another 30 years until we were able to put the major parts of ferroptosis jigsaw together. For this we can be grateful to the work of the Dr. Stockwell's and Dr Conrad's groups, done in the 10-year long period from 2001 to 2012.

What we have learned about ferroptosis during the decade that followed? Ferroptosis is classified under "regulated" types of cell death relying on dedicated molecular machinery, and as such, can be induced/prevented by different pharmacological/genetic manipulations. On the other side, it is still not clear whether ferroptosis can be classified in the group of "programmed" cell deaths, considering that in contrast to the 'clean'apoptosis cell death, ferroptosis leads to a sort of explosive necrotic cell death able to induce an inflammatory response.

The molecular machinery dedicated to ferroptosis ${ }^{24-26}$ has been depicted in the Fig. 1 with the detailed description in the figure legend. In short, in the homeostatic conditions, enzymatically or non-enzymatically 


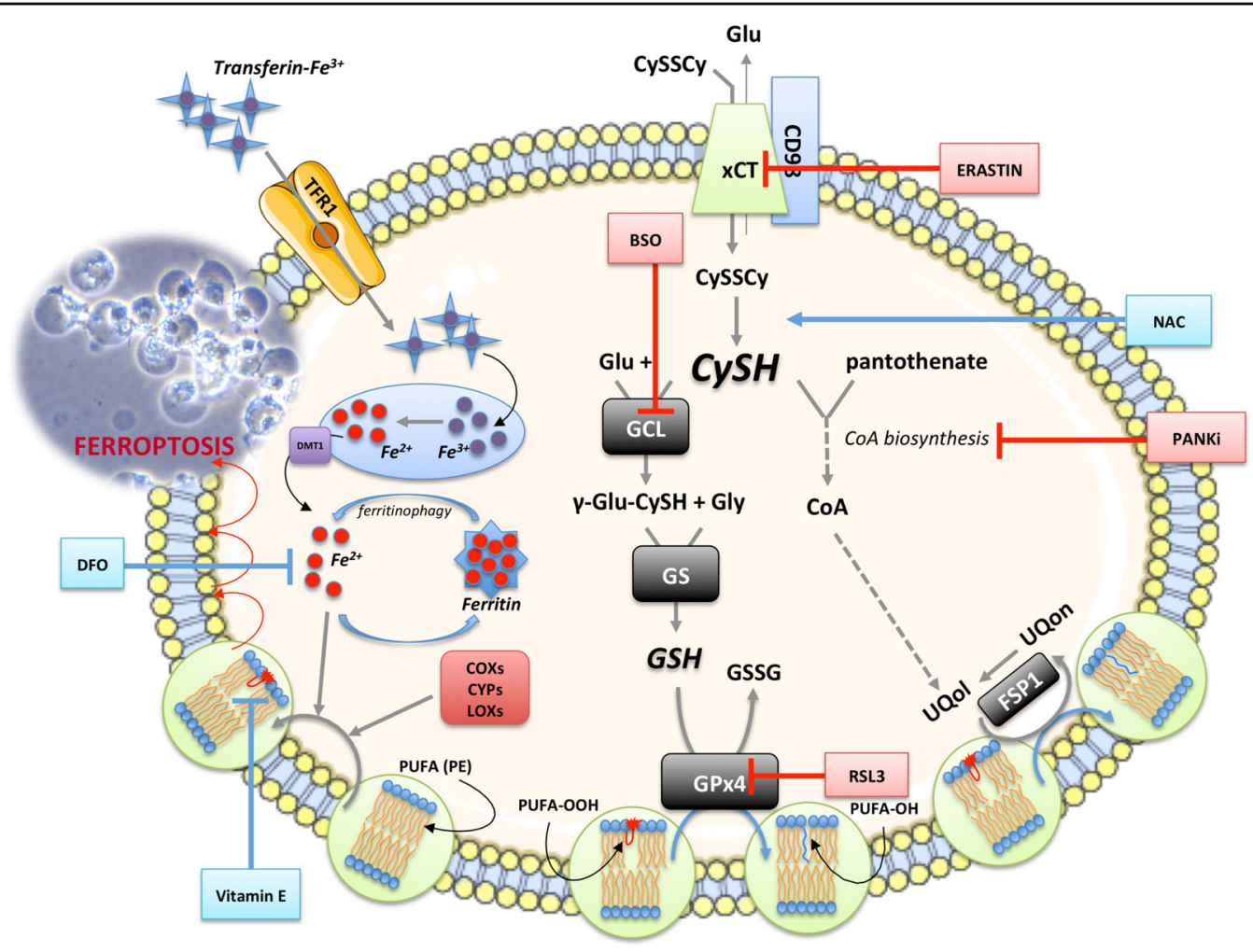

Fig. 1 Ferroptosis overview. Under basal conditions, cancer cells take up cystine (CySSCy) via xCT transporter, reduce it and use it for many different purposes. One of the main roles of CySH in the cell is synthesis of glutathione (GSH). GSH serves as co-substrate for many antioxidant enzymes, including glutathione peroxidase 4 (GPx4). In the context of ferroptosis, the GPx4 plays an important role as neutralizer of oxidative damage in the membrane compartments of the cell. In the presence of 'labile' $\mathrm{Fe}^{2+}$ ions (Fenton reaction), oxidants attack membrane polyunsaturated fatty acids (PUFA), such as phosphatidyl-ethanolamine (PE), converting them to highly toxic lipid peroxides (PUFA-OOH). Due to its high redox potency, the level of iron in the cell is kept under tight regulation. Extracellular $\mathrm{Fe}^{3+}$, bound to transport protein transferrin, is taken up by cells via transferrin receptor 1 (TFR1), transported into endosome where it undergoes reduction to $\mathrm{Fe}^{2+}$ by metalloreductases. Divalent metal transporter 1 (DMT1) mediates the transport of $\mathrm{Fe}^{2+}$ from the endosome into the cytoplasmic labile iron pool, where most of it is ligated by heme, bound in FeS clusters, or stored in the iron storage protein ferritin. However, small amount of free and catalytically active $\mathrm{Fe}^{2+}$ ions is present in the cytoplasm coming either directly from endosomes or from autophagic degradation of ferritin in the process known as ferritinophagy. In addition to being formed through non-specific propagation of radicals, oxidized lipids can also be synthesized in an enzymatically-regulated manner by cyclooxygenases (COXs), cytochrome p450s (CYPs), and lipoxygenases (LOXs). Under basal conditions, the level of PUFA-OOH is controlled by the action of GPx4 enzyme. This Se-peroxidase uses reducing power of GSH to convert toxic PUFA-OOH into non-toxic alcohol form (PUFA-OH), protecting membrane and cellular integrity. Alternatively, PUFA-OOH can be reduced by the ubiquinol (UQol). Thereby produced ubiquinon (UQon) is reduced back to UQol form by the action of ferroptosis suppressor protein 1 (FSP1). Blocking CySSCy import via xCT (erastin), synthesis of GSH (buthionine sulfoximine, BSO), GPX4 activity (RAS-selective lethal 3, RSL3) or the biosynthesis of UQol (PANKi), antioxidant balance within the cell is disturbed and

accumulation of lipid peroxide leads to the lost of plasma membrane assembly, composition, structure, and finally cell osmotic necrotic collapse and death (ferroptosis). This type of the cell death is classified under 'regulated' forms of cell death as some compounds can specifically prevent it. As an example, Vitamin E (tocopherol a) a lipophilic antioxidant that can incorporate to the membrane compartment and stop chain-formation of PUFA$\mathrm{OOH}, \mathrm{N}$-acetylcysteine (NAC) can serve as an alternative source of cysteine, while deferoxamine (DFO) chelate 'labile' metal ions including Fe ${ }^{2+}$. This figure was created using Servier Medical Art templates, which are licensed under a Creative Commons Attribution 3.0 Unported License;

https://smart.servier.com.

produced membrane lipid peroxides are effectively reduced to non-toxic alcohol derivatives, by the action of the Se-containing GPx4 enzyme ${ }^{21}$. The reducing power that drives the regeneration of GPx4 is GSH, a major nonenzymatic antioxidant in the cell ${ }^{27}$. Cellular concentration of GSH largely depends on the rate-limiting step in its biosynthesis catalysed by glutamate-cysteine ligase (GCL), or more precisely on the availability of the rate-limiting amino acid - $\mathrm{CySH}^{28}$. From its side, cysteine can be synthesized within the cell from methionine via transsulfuration pathway ${ }^{29}$. However, previous studies showed that this does not meet the requirements of highly proliferative and/or oxidatively compromised cells (such as cancer cells), which thus, largely rely on the import of this amino acid from the extracellular space $^{30}$. Accordingly, the major transporter for the oxidized form (dominant 
form in the serum and almost exclusive form in the culture media) of $\mathrm{CySH}$ (cystine, CySSCy), known as Xcsystem, seems to be consistently up-regulated within different types of cancer ${ }^{31-40}$. Xc- system, composed of a light transporter chain $(\mathrm{xCT}, S L C 7 A 11)$ and a heavy chaperon subunit (CD98, SLC3A2), is an obligatory exchanger, allowing the import of CySSCy at the expense of glutamate export (1:1) (reviewed in ref. ${ }^{41}$ ).

\section{Cell-born mechanisms of the resistance to ferroptosis induction}

Until recently, the $\mathrm{xCT}-\mathrm{GSH}-\mathrm{GPx} 4$ axis has been seen as indispensable for prevention of ferroptosis. Hence, blocking CySSCy import via $\mathrm{xCT}$ (i.e. by erastin), synthesis of GSH (i.e. by buthionine sulfoximine, BSO) or GPx4 activity (i.e. by RAS-selective lethal 3, RSL3) proved to induce accumulation of membrane lipid peroxides within different cancer cell types, further inducing the loss of membrane assembly, structure, dynamics, and finally, cell death (ferroptosis) ${ }^{42,43}$. However, very recent studies point out that GSH-GPx4 part of the axis could be dispensable (reviewed in ref. ${ }^{44}$ ), thanks to the presence of ubiquinol, as a reducing agent, and ferroptosis-supressor protein 1 (FSP1), as its regenerating enzyme, in the membrane compartments of the cell ${ }^{45,46}$. Even more, some additional roles (besides involvement in GSH biosynthesis) of $\mathrm{CySH}$ have been suggested as important for the process of ferroptosis, such as its incorporation into Coenzyme A-precursor of ubiquinol, via pantothenate pathway ${ }^{44,47}$, as well as its role in protein folding and endoplasmic reticulum homeostasis ${ }^{48,49}$. Lastly, an important and frequently overlooked feature of the GPx4 enzyme is its broad substrate specificity. Previous studies showed that GPx4 could efficiently use other thiol compounds (cysteine, among them) as a reducing power, instead of GSH, which can explain relative insensitivity of the cancer cells to GSH depletion ${ }^{50-53}$. In line with this is the study of Banjac and co-workers who demonstrated that lipid peroxidation can easily be prevented, even in the GSH-depleted conditions, thanks to the $\mathrm{xCT}$-driven cystine/cysteine cycle ${ }^{54}$. In order to evaluate the contribution of these cell-born alternative pathways in the prevention of overwhelming accumulation of the lipid peroxides in the cell, additional studies are needed.

Although alternative cystine transporter (known as BAT1) exists ${ }^{55,56}$, to the best of our knowledge, its role in ferroptosis induction still has not been demonstrated, leaving the $\mathrm{xCT}$ alone in this context. The antiporter system $\mathrm{Xc}^{-}$indeed seems to be indispensable transporter for CySSCy; however, studies suggest that the reduced form of this amino acid plays an important role in the resistance to $\mathrm{xCT}$-dependent ferroptosis in vivo ${ }^{34,57}$. Although $\mathrm{CySH}$ in these conditions can be provided from the circulating blood, a growing number of studies suggest that the main source of cysteine and/or GSH to cancer cells are neighbouring cells. Interestingly, it is also suggested that cell-to-cell contact and its downstream signalling alone are able to induce ferroptosis resistance in the cancer cells, independently of cysteine-delivery. All of these aspects are discussed in more details below.

\section{Cell-to-cell contact}

The importance of cell density for the sensitivity of mammalian cells to cysteine-starvation has been recognized as early as in the mid- $20^{\text {th }}$ century, when Harry Eagle noticed that the cells become highly sensitive to cysteine removal, unless cell density reaches the "appropriate level" ${ }^{58}$. Later on, when the role of $\mathrm{xCT}$ was revealed, the same conclusion had been drawn once again in the comprehensive review article of Lewerenz and coworkers ${ }^{41}$. Interestingly, even in the case of GPx4 enzyme, it has been noted that GPx4-KO mouse embryonic fibroblasts are able to grow if seeded at higher density or in 3D spheroids, but not if seeded sparsely ${ }^{22,59}$.

Increased cell density is notably related to the resistance of solid tumours to many different chemotherapeutics, and in most cases, this has been linked to G1 cell cycle elongation time/arrest ${ }^{60-64}$. However, recent studies suggested that the density-related resistance might be a consequence of other molecular events, independently of the $\mathrm{G} 1$ cell cycle arrest ${ }^{65-68}$. This seems to be also the case for cysteine-starvation resistance, as the effects of increased density have been observed even before complete confluence (and arrested proliferation) is reached $^{34,69}$. Also, Wenz and collaborator showed that density-related resistance to ferroptosis induced by tertbutyl hydroperoxide has not been a simple consequence of the growth arrest ${ }^{69}$.

\section{Hippo pathway involvement in the ferroptosis resistance: the role of YAP/TAZ}

First papers dealing with the mechanistic insights of density-based resistance to ferroptosis appeared quite recently and suggested the Hippo pathway as a major player this event ${ }^{70-74}$. In brief, the main role of the highly conserved Hippo pathway is to restrict tissue growth in adults and to modulate cell proliferation, differentiation, and migration in developing organs, in response to different signals including cell-to-cell contact, cell polarity, energy status, mechanical clues, etc. The core of the Hippo signalling pathway consists of: the mammalian Ste20-like kinases 1/2 (MST1/2), large tumour suppressor $1 / 2$ (LATS1/2), as well as two transcriptional co-activators: yes association protein (YAP) and its paralog WW domain containing transcription regulator 1 (TAZ). When activated, Hippo phosphorylation cascade starts by activation of MST1/2, which then, in cooperation with neurofibromin 2 (NF2 aka merlin), recruits and activates 

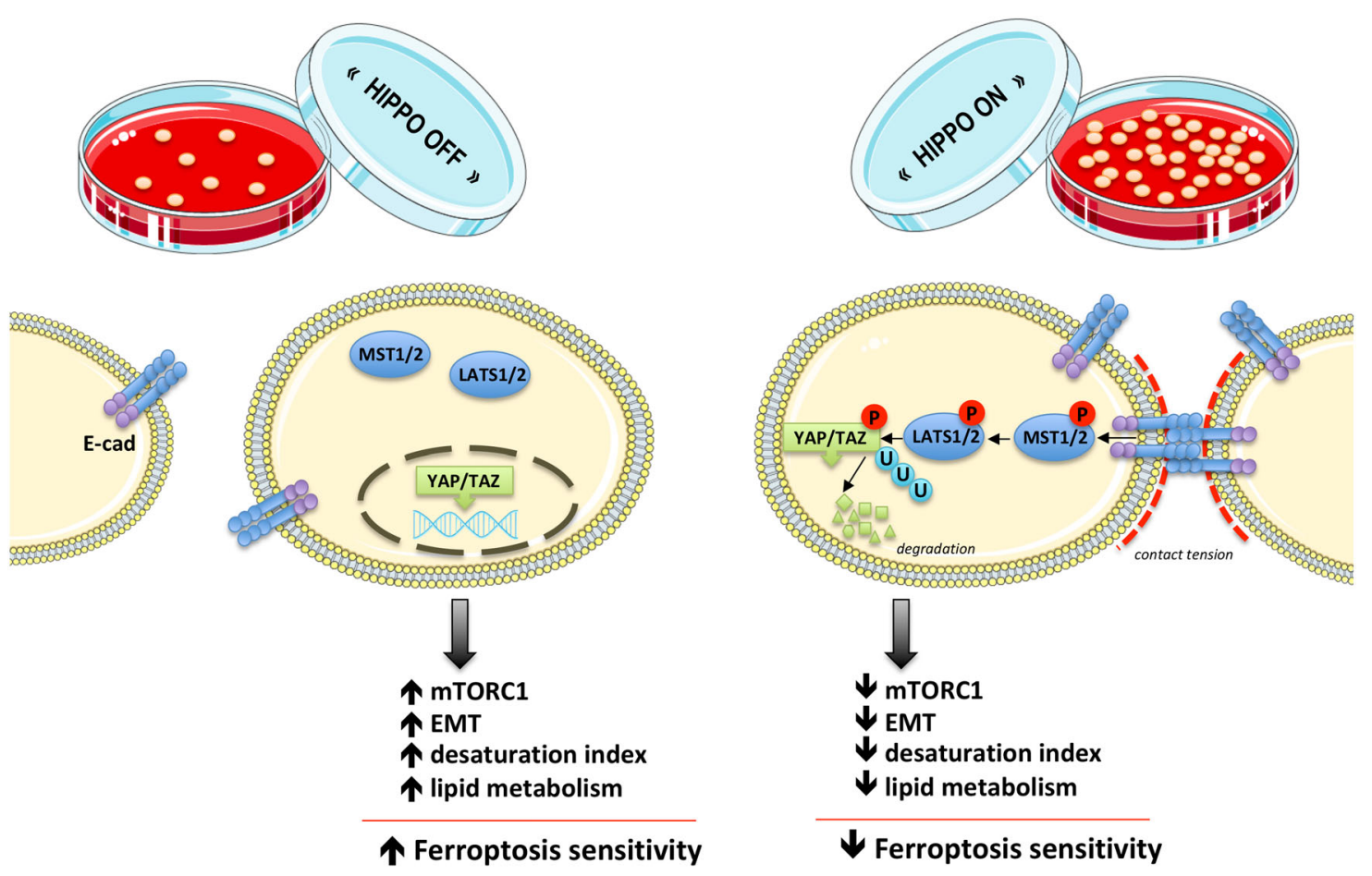

Fig. 2 The effect of Hippo pathway on the sensitivity of cancer cells to ferroptosis. Cell-to-cell contact or other mechanical clues activate phosphorylation cascade of Hippo pathway that starts with mammalian Ste20-like kinases 1/2 (MST1/2), further activating large tumour suppressor $1 /$ 2 (LATS1/2) and resulting in phosphorylation, nuclear extrusion and degradation of two transcriptional co-activators: yes association protein (YAP) and its paralog WW domain containing transcription regulator 1 (TAZ). The effects of this molecular cascade branch to many intracellular signalling pathways (as described in the text) and ultimately result in decreased sensitivity of cancer cells to ferroptosis. This figure was created using Servier Medical Art templates, which are licensed under a Creative Commons Attribution 3.0 Unported License; https://smart.servier.com.

LATS1/2. From its side, LATS1/2 phosphorylates and inactivates YAP/TAZ, leading to their nuclear extrusion ${ }^{75}$. Moreover, it has been suggested that YAP/TAZ phosphorylation by LATS1/2 makes these transcriptional co-activators more prone to ubiquitination and degradation ${ }^{76,77}$.

The work of $\mathrm{Wu}$ and colleagues ${ }^{70,71}$ showed that the different sensitivity of a panel of human epithelial cancer cell lines to ferroptosis correlates with E-cadherin expression and Hippo pathway activity, seen through increased phosphorylation and decreased nuclear localization of YAP. The data also demonstrated that E-cadherin, NF2 or LATS1/2 knockdowns, have the same effect as low density, i.e. increase sensitivity to ferroptosis, while YAP(S127A), the mutant that cannot be phosphorylated by LATS1/LATS2, exhibited opposite effect even at a higher density or in spheroids. This further provides evidence for the functional link between E-cadherin, Hippo pathway and ferroptosis. Another study done on the highly ferroptosis-sensitive renal cell carcinoma (RCC) came to the same functional link, although focusing on the other Hippo pathway regulator-TAZ ${ }^{73}$.

Although mutation in the Hippo components are uncommon in tumours, increasing number of data point out the importance of Hippo pathway (de)regulation in the cancer, which most likely stems out from tight interconnections between this and other crucial cellular pathways ${ }^{75}$. In the next four subsections, we will propose how these intricate signalling pathways might elicit resistance to ferroptosis (summarized in Fig. 2). However, it is very important to point out here and to keep in mind for the future research that the role of the Hippo pathway can be seemingly applied only to adherent cell, while its role for the hematopoietic cell (patho)physiology seems to be dispensable ${ }^{78}$. On the other side, the importance of the cell density for the growth and survival of lymphoma cells has been shown, just as their dependency on the proper thiol supply in the low-density conditions ${ }^{79}$. This clearly suggests that in the case of the cells in suspension, some other/additional mechanisms might be involved, and that the effects of the cell density on the cancer cell sensitivity to ferroptosis might go well beyond the Hippo pathway.

\section{Hippo pathway and lipid peroxides}

One of the major hallmarks of ferroptosis is the accumulation of membrane lipid peroxides. The main targets for this type of oxidative insult are polyunsaturated fatty acids (PUFAs) of membrane phospholipids containing 
bis-allylic hydrogen atoms that can be readily abstracted ${ }^{80}$. Hence, abundance of PUFAs largely influences the rate of peroxidation, and consequently, ferroptosis sensitivity. It has been shown that knockout of the genes involved in the biosynthesis and remodelling of PUFAs: long-chain-fatty-acid-CoA ligase 4 (ACSL4) or lysophosphatidylcholine acyltransferase 3 (LPCAT3) significantly decreases the sensitivity toward ferroptosis ${ }^{81-84}$. Interestingly, YAP/TAZ seem to be involved in the process of desaturation of the fatty acids. Specifically, stearoyl coenzyme A desaturase 1 (SCD1), an enzyme involved in the conversion of saturated into unsaturated fatty acids, mediates the release of YAP/TAZ from the $\beta$-catenin destruction complex. The data from lung adenocarcinoma samples revealed that SCD1 co-expresses with nuclear YAP/TAZ suggesting a clear correlation between expression and activity of these genes, and thus with the desaturation index of the cell $^{85-87}$. Although this link between YAP/TAZ-dependent unsaturation index of the cell and ferroptosis sensitivity has still not been investigated, studies showing that E-cadherin-dependent YAP/ TAZ nuclear extrusion decreases the accumulation of lipid peroxides ${ }^{69-71,73}$ suggest that this is something that deserves further and more in depth analysis.

Another way how Hippo components can affect the degree of lipid peroxidation is directly through influencing the ROS-producing systems. In the report of Yang and co-workers it has been shown that active and nuclearlocalized TAZ regulates epithelial membrane protein 1 (EMP1) expression, which in turn increases NAD(P)H oxidase 4 (NOX4) expression in RCC. NOX4 is a ROSproducing enzyme whose involvement in lipid peroxidation has been noted in the previous studies ${ }^{73,74}$. An interesting observation of this study is that erastin did not affect GSH level in the TAZ knockout cells expressing low level of NOX4 (but did in WT cells with higher amount of NOX4), thus indicating that most of GSH reducing power in RCC has been used for amelioration of the NOX4dependent oxidative damage ${ }^{73}$. Considering that NOX4 is an important source of ROS in the kidney ${ }^{88,89}$, the question that remains: is TAZ-EMP1-NOXs axis typical of renal redox homeostasis, and if so, are there other ROSgenerating enzymes that might serve the same purpose in other tissues?

\section{Hippo pathway and EMT}

Long-lasting controversy about the importance of epithelial-to-mesenchymal transition (EMT) in cancer dissemination and metastasis in vivo, got its final proof relatively recently with epithelial lineage tracing in a mouse model ${ }^{90}$. On the other side, the role of EMT in cancer chemoresistance has been a widely accepted paradigm $^{91}$. Due to high selective pressure, cells undergoing EMT have, from one side, low survival success rate, but from another, the ones that survive, do so by acquiring the resistance to many different environmental insults, including chemotherapy ${ }^{91,92}$. One of the rare studies challenging this dogma is the report of Viswanathan and colleagues, who showed that the therapyresistant, highly mesenchymal cells are dependent on the $\mathrm{xCT}-\mathrm{GSH}-\mathrm{GPx} 4$ axis for their redox homeostasis, and are thus, extremely sensitive to ferroptosis ${ }^{93}$. Similar result has been obtained in our previous study, where sensitivity of epithelial pancreatic ductal adenocarcinoma (PDAC) cell line (Capan-2) to erastin increased upon induction of EMT with tumour growth factor $\beta$ (TGF- $\beta$ ) treatment ${ }^{34}$. Although the higher sensitivity of mesenchymal phenotype to ferroptosis may look contra-intuitive at a first glance, when placed in the context of what has been aforementioned about cell-to-cell contact and resistance to ferroptosis, it makes much more sense.

One of the major characteristics of EMT is the loss of cell-to-cell contact and decreased expression of the canonical epithelial marker-E-cadherin ${ }^{94}$. Numerous studies dealing with the connections between Hippo pathway and EMT showed that overexpression of the Hippo pathway regulators, YAP/TAZ, is tightly related to the induction of the EMT ${ }^{95-99}$. Even more, some data points out the collaborative nature of the YAP/TAZ and EMT transcriptional factors ${ }^{100}$. Considering that PUFAs are essential for membrane fluidity, it is logical to assume that increased unsaturation index increases during EMT, when epithelial cells lose polarity and cell-cell adhesion and acquire mesenchymal morphology. Indeed, it has been observed that overexpression of enzymes such as ACSLs and SCD1 (involved in lipid metabolism and increased unsaturation index) induces EMT and increases migration/invasion of colorectal cancer cells ${ }^{101}$. Considering the already mentioned connection between Hippo pathway and unsaturation index, it seems plausible that this represent yet another way how the loss of cell-tocell contact exposes cell vulnerability to lipid peroxidation, and consequently, ferroptosis. In this context, it might also be interesting to evaluate the possible interplay between hypoxia and the Hippo pathway, as hypoxiainducible factor 1 (HIF-1) has been recognized as a key factor stimulating EMT and ferroptosis ${ }^{102-105}$.

According to all of this, it seems that the induction of EMT can deprive cells from protective mechanisms of cell-to-cell contact. However, the situation is not that simple. Indeed, Panzilius et al. ${ }^{106}$ recently observed that the overexpression of EMT transcription factor Twist1 or Snail1 was not sufficient to overcome density-related resistance in the human mammalian epithelial cells, although classical markers of mesenchymal phenotypes were detected. This might suggest that the signals induced by EMT transcription program alone are not sufficient to overcome density-induced resistance in all cases. 
However, it is worth noting that in this study the effects of EMT induction on GPx4 inhibition (ferroptosis) were not detected at any cell density, although the amount of this enzyme was 2.3-2.6 times higher in Twist1-expressing cells. Obviously, much more in depth analysis regarding the Hippo-(in)dependent EMT effects on ferroptosis sensitivity has to be undertaken in order to clarify whether, how and to what extent this pathway can influence ferroptotic process.

One very important aspect worth mentioning here and keeping in mind for the future research is that EMT, during cancer cell migration, does not necessary need to be complete. Namely, studies done by Jolly and collaborates ${ }^{107}$ showed that the migration and invasion of the cancer cells can be achieved through partial EMT. The clusters of cancer cells undergoing this non-compete EMT do partial de-differentiation while still maintaining cell-to-cell contacts (reviewed in refs. ${ }^{108,109}$ ). Considering the topic of this review and all that had been said about sensitivity to ferroptosis in the context of cell-to-cell contacts, this study is of utmost importance for understanding relation between cell-to-cell contact, EMT and ferroptosis sensitivity.

\section{Hippo pathway and mTORC1}

Hippo and mechanistic target of rapamycin complex 1 (mTORC1) pathways are the two dominant regulators of normal organ growth and development. Although seemingly interconnected, the very crosstalk between them has not been fully illuminated. More precisely, different intersection points of this bidirectional regulation have been reported up to now. Thus, for both mTORC1 and mTORC2, it has been shown to positively regulate YAP stabilisation and signalling ${ }^{110-112}$, while different components of the Hippo pathway have been marked as regulating points of mTORC1. A recent report of Gan and coworkers $^{113}$ showed that LATS1/2 suppresses mTORC1 activity by phosphorylation of S606 Raptor, and consequently preventing Raptor-Rheb interconnection. Similarly, NF2-deficient human meningioma cells and NF2-KD arachnoidal cells show rapamycin-sensitive constitutive mTORC1 activation ${ }^{114}$. On the other side, YAP/TAZ components of the Hippo pathway stimulate mTORC1 activity either through downregulation of PTEN (upstream negative regulator of mTORC1) or via upregulation of LAT1 (transporter of essential amino acid, necessary for mTORC1 activation) expression ${ }^{115-117}$.

Corroborating with this, our study showed, as mentioned previously, that highly epithelial PDAC cell lineCapan-2, has shown higher resistance to erastin-induced ferroptosis, both in term of kinetic and concentration, in comparison to mesenchymal MiaPaCa-2 PDAC cell line ${ }^{34}$. However, induction of EMT by TGF- $\beta$ treatment equalized the sensitivity of these two PDAC cell lines.
Interestingly, one of the sticking effects observed was the increase of mTORC1 activity upon TGF- $\beta$ treatment, seen through increased phosphorylation status of mTORC1 pathway components. Here, we speculate that effects of TGF- $\beta$ are the results of an enhanced protein synthesis and metabolism in general. Accordingly, protein synthesis inhibition by cycloheximide decreased sensitivity of MiaPaCa- 2 cells to ferroptosis. Very similar results have been observed by Dixon and co-workers who, using livecell time-lapse imaging, screened more than 1800 small molecules for ferroptosis and apoptosis inducers ${ }^{118}$. According to the data, ATP-competitive mTORC1, and mTORC1/PI3K inhibitors were associated with ferroptosis resistance; however is important to note that this was true only in the case of $\mathrm{xCT}$-dependent ferroptosis, while no effects have been observed when GPx4 inhibitors were used for ferroptosis induction. Most likely, the explanation lies in the fact that mTORC1 inhibition decreases flux of amino acid into proteins and consequently redirects cysteine into GSH biosynthesis, which is confirmed by increased GSH content in the cells that were treated with $\mathrm{mTORC} 1$ or protein synthesis inhibitors ${ }^{118}$.

Although suppression of mTORC1 indeed seems like something that might be a problem in the cancer treatment context, it seems that the opposite is true for nontransformed cells. Namely, it has been shown that mouse cardiomyocytes isolated from cardiac-specific mTOR transgenic mice are less sensitive to Fe-donor, erastin, and RSL3-induced cell death in comparison with the control, while the opposite is observed in the case of cardiomyocytes isolated form cardiac-specific mTOR knockout mice $^{119}$. This discrepancy in the effects of mTORC1 inhibition to ferroptosis sensitivity observed between transformed and non-transformed cells might be rooted in the well-known deregulation of mTORC1 activity upon neoplastic transformation. However, further studies are needed to clarify this issue.

\section{Hippo pathway and proteotoxic stress}

In the section where cell-born resistant mechanisms have been discussed, we briefly mentioned a very interesting study published recently by Harris and collaborators who showed that cancer cells display a wide range of sensitivity toward GSH depletion ${ }^{49}$. Although not directly stated in the paper, this suggests that GSH might be dispensable, and thus not the best target for ferroptosis induction in cancer. Using both pharmacological and genetic screening approaches the authors revealed the activity of some deubiquitinases as being central for the maintenance of protein homeostasis and thus survival upon GSH depletion. Similarly, Dixon et al. $^{48}$ showed that ER stress and disturbed protein homeostasis are a very important part in $\mathrm{xCT}$-dependent initiation of ferroptosis. In both cases, it has been 
suggested that cell death occurs as a consequence of deregulated antioxidant defence (GSH depletion), followed by proteotoxic stress (due to cysteine starvation-induced unfolded protein response/ER stress or deubiquitinase inhibition).

No connection in the literature have been made between this proteotoxicity-induced ferroptosis and cell-to-cell contact, but it is worth noting that the activity of Hippo pathway depends largely on ubiquitination-deubiquitination status (review in ref. ${ }^{120}$ ). Namely, the activities of Hippo pathway components, including YAP/TAZ regulators, are under direct regulation of ubiquitination-deubiquitination process. Hence, it would be of great importance to illuminate how proteotoxic stress and disbalance in the ubiquitination-deubiquitination process may influence cellto-cell physiology, and vice versa, especially in the case of epithelial cells resistant to $\mathrm{xCT}$-dependent ferroptosis.

\section{Effect of cell-to-cell contact on the cancer metabolic rewiring}

Metabolic rewiring due to the loss of cell-to-cell contact is mainly studied in the context of EMT and cancer metastasis $^{121}$. Although the effects of these changes on ferroptosis sensitivity have not been extensively investigated, they might be of particular importance, especially as many already FDA-approved drugs targeting cancer metabolism could significantly improve the effectiveness of ferroptosis inducers. In the previously mentioned study of Panzilius et al. ${ }^{106}$, the authors showed that cell density significantly increases catabolism of lipids at the expense of increased vulnerability toward ferroptosis. More precisely, low density induces the release of free fatty acids from the lipid droplets, which further fuel ATPproduction through mitochondrial $\beta$-oxidation. Hence, sensitivity to pharmacological/genetic invalidation of GPx4 in sparsely seeded human mammalian epithelial cells was reverted by inhibition of the adipose triglyceride lipase (ATGL) - the first enzyme in triacylglyceride hydrolysis, or by inhibition of $\beta$-oxidation. Similar effect has been observed in the case of mono-unsaturated fatty acid oleic acid treatment, which increases the content of cellular lipid droplet ${ }^{122}$. Taking into account what has been said about mTORC1 changes upon cell-to-cell contact lost, as well as its recognized, central role in lipid metabolism (for review see ref. ${ }^{123}$ ), it is reasonable to assume that some of these metabolic changes stems from mTORC1 signalling, and consequently, could be manipulated upstream.

\section{Cell-to-cell interplay}

Tumour microenvironment has long been recognized as an important factor in cancer progression and response to chemotherapy ${ }^{124}$. The specificities of this microenvironment significantly vary from one to another cancer type and generally depend on the types of surrounding non-transformed cells (fibroblasts, vascular endothelial cells, immune cells, etc.), as well as the characteristics of the extracellular matrix and milieu in which both transformed and non-transformed cells are deeply immersed (stiffness, oxygen, nutrient level, $\mathrm{pH}$, etc.). The interaction between cancer and stromal cells is always bidirectional. Hence, although generally marked as "non-transformed", stromal cells are significantly different to their counterparts in non-transformed tissues and organs. One of the characteristic examples are cancer-associate fibroblasts (CAFs), which seem to play an important role in cancer cell proliferation, invasion, and many other tumour-promoting activities (reviewed in ref. ${ }^{125}$ and ref. ${ }^{126}$ ), and their presence and activity in many different tumours correlates with patient poor prognosis ${ }^{127}$. Hence, it did not come as a surprise that stromal cells significantly influence response of cancer cells to ferroptosis induction as well.

Interesting data coming from the report of Wang and colleagues ${ }^{128,129}$ showed that resistance of breast cancer cells to platinum-based drugs comes from close interconnection between cancer cells and fibroblasts. Namely, cisplatin induces a severe drop in GSH level (most likely due to the mechanisms of cisplatin efflux from the cells that require its chelation with GSH), and thus, compromised survival of ovarian cancer cells ${ }^{130}$, which might explains the initial response of ovarian cancer to the therapy. However, this was easily prevented when cancer cell were co-cultured with fibroblasts. According to the data, fibroblasts were able to import oxidized (dominant) form of cysteine from the extracellular space, to reduce and make it available to cancer cells (Figs. 3, 4). This phenomenon might explain the in vivo resistance of highly (in vitro) sensitive $\mathrm{xCT}-\mathrm{KO}$ cells shown by our group and others ${ }^{34,57}$. This CySH/GSH shuttle between $\mathrm{xCT}$-expressing fibroblasts and cancer cells sounds rather logical, as knockdown of $\mathrm{xCT}$ in fibroblasts prevent fibroblast-mediated cisplatin resistance in ovarian can$\operatorname{cer}^{129}$. Even more, it seems that interferon- $\gamma$ (IFN- $\gamma$ ) secreted from $\mathrm{CD}^{+} \mathrm{T}$ cells, has the same effect by inducing fibroblast-specific downregulation of $\mathrm{xCT}^{129}$. This is very similar concept to cystine/cysteine cycle observed in Burkitt's lymphoma cells overexpressing $\mathrm{xCT}$, which allow them to maintain the redox homeostasis even under GSH-depleting conditions ${ }^{54}$. However, Arensman and co-workers showed that resistance of $\mathrm{xCT}-\mathrm{KO}$ cells is maintained even if the xenografts are implemented into $\mathrm{xCT}-\mathrm{KO}$ mice $^{57}$, raising the question whether $\mathrm{xCT}$ mediated uptake of CySSCy and subsequent $\mathrm{CySH} / \mathrm{GSH}$ shuttle is the only mechanism conferring ferroptosis resistance in the stromal compartment in vivo. A recent study of Zhang et al. ${ }^{131}$ suggests an alternative mechanism by which fibroblasts suppress ferroptosis and enhance 


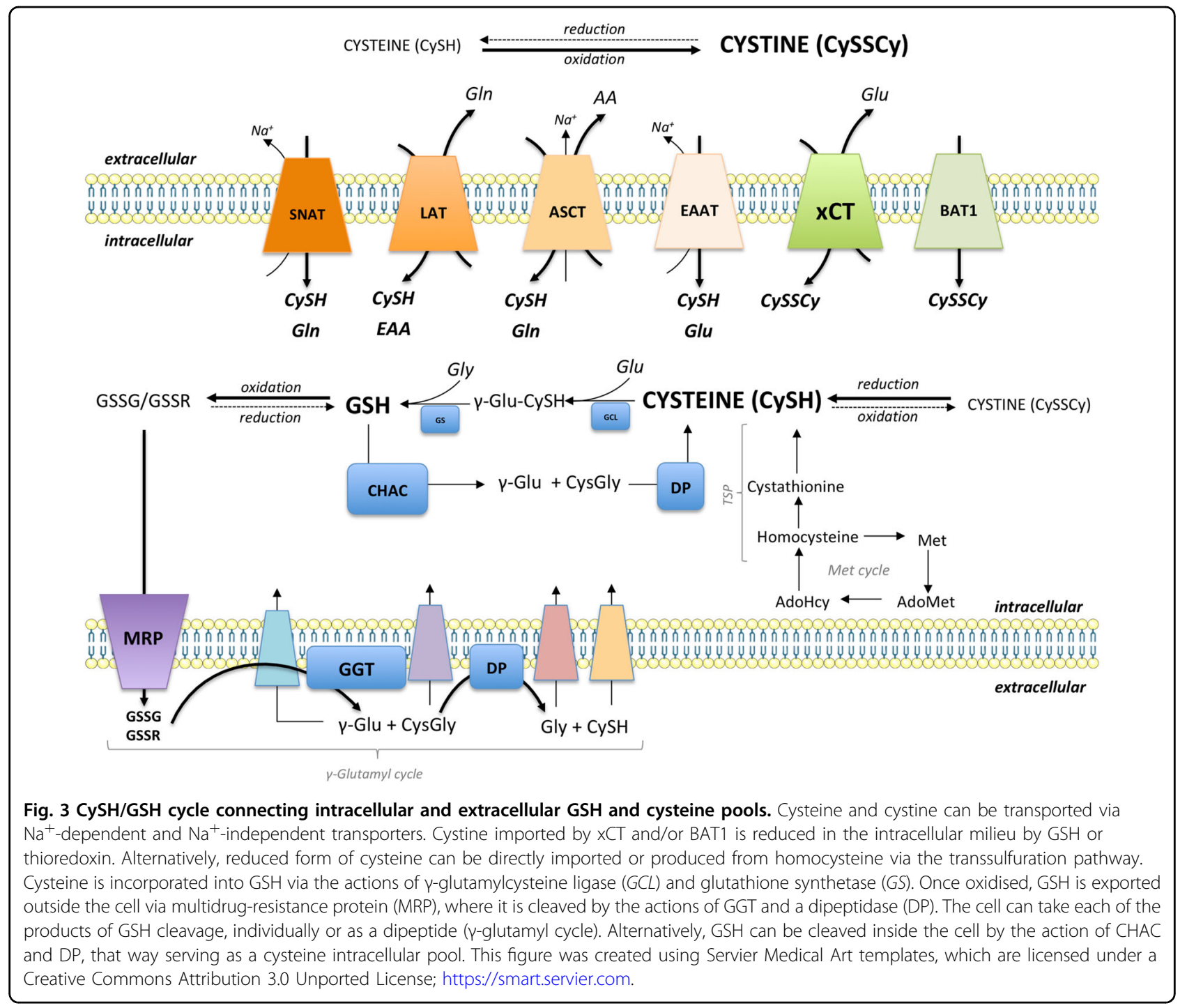

chemoresistance in cancer cells: the secretion of miR-522 that leads to the suppression of arachidonate lipoxygenase 15 (ALOX15), an enzyme involved in lipid peroxidation. Altogether, the results suggest that the metabolic, but also cytokine, secretome of the stromal cells has to be taken in consideration when ferroptosis resistance is discussed.

Indeed, a key aspect of cell-to-cell interplay in stromal compartment is the interaction of immune and cancer cells. As mentioned previously, secretion of INF- $\gamma$ by $\mathrm{T}$ cells has been suggested as ferroptosis stimulator in the case of ovarian cancer ${ }^{128,129}$. Interestingly, another way around has been shown as well. Nomi et al. ${ }^{132}$ observed that despite in vivo PDAC cells show resistance to $\mathrm{xCT}$ induced ferroptosis, the genetic invalidation of $\mathrm{xCT}$ makes these cells more susceptible to immunotherapy. Namely, the clinical trials with antibodies against the two main checkpoint targets: programmed associate protein 1 (PD-1) and PD-1 ligand (PD-1L), did not show any benefit for pancreatic cancer patients, although PD-1L expression has been associated with poor prognosis in such patients ${ }^{132}$. The reason for this is still elusive, but a possible explanation may lay in the low immunogenic nature of PDAC cells due to the low mutation burden ${ }^{133}$. Interestingly, $\mathrm{xCT}-\mathrm{KO}$ in pancreatic, but also colon cancer cell lines, enhanced the efficacy of checkpoint immunotherapy in a mouse xenograft model $^{57}$, and it has been explained by a cysteine starvation-induced ER stress, which is necessarily connected with increased cell immunogenicity $^{48,134,135}$.

\section{Conclusion}

Almost a century-long fundamental research contributed to the emergence and contextualization of a new type of cell death-ferroptosis. At the very beginning of ferroptosis research, as we know it today, the mechanisms sounded very simple in comparison with the intricate 


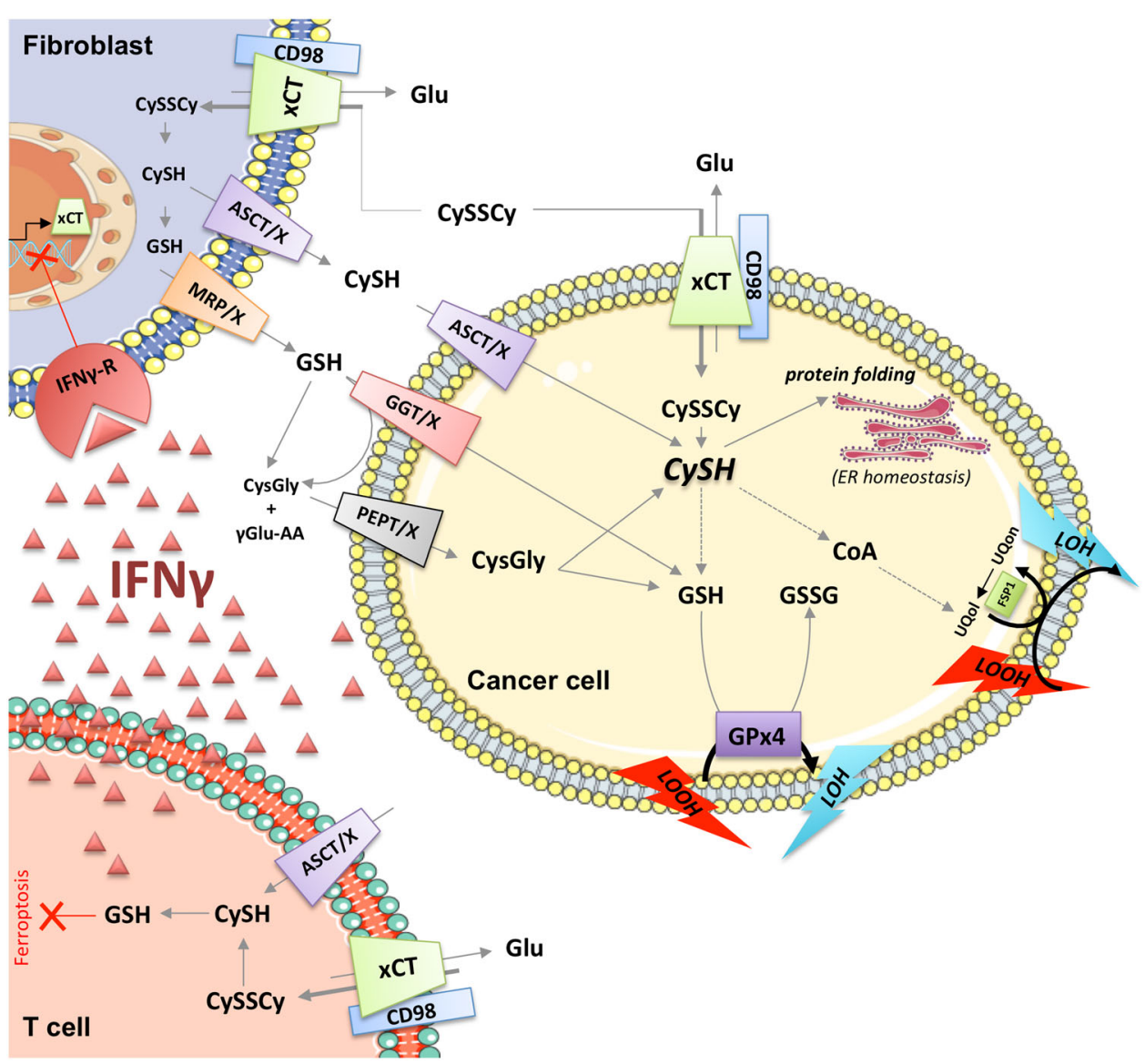

Fig. 4 Cell-to-cell interplay (explained in the text). Glu, glutamate; CySH, cysteine; CySSCy, cystine; GSH, reduced glutathione; GSSG, oxidized glutathione; CoA, coenzyme A; UBol, ubiquinol; UBon, ubiquinone; FSP1, ferroptosis-suppression protein 1; LOOH, lipid peroxides; LOH, lipid alcohols; xCT, light chain of the system XC- (cystine-glutamate transporter); CD98 chaperon heavy chain of the system Xc-; ER, endoplasmic reticulum; CysGly, cysteinyl-glycine; ASCT, alanine-serine-cysteine transporter; GGT, gamma-glutamyltranspeptidase; PEPT, peptide transporter; $X$, unknown transporter; YGlu-AA, gamma-glutamyl-amino acid; IFNy, interferon gamma; IFNYR, IFNy receptor. This figure was created using Servier Medical Art templates, which are licensed under a Creative Commons Attribution 3.0 Unported License; https://smart.servier.com.

signalling machinery employed for a defined apoptotic execution. However, almost ten years in, and we already can say that the situation with ferroptosis is not that simple as we might have thought. One of the most striking things that have been observed since the beginning, with now a better overall understanding of the underlying mechanisms, is the remarkable difference in the ferroptosis sensitivity between the cells seeded at different density. Understanding the physiology of cell-tocell contact and how it provides resistance to oxidative insults, like lipid peroxidation, can tell us much more about the chemotherapeutical regimens that have to be used for maximal efficacy of ferroptosis inducers.

\section{Acknowledgements}

S.C. and W.M. are supported by a post-doctoral fellowship from GEMLUC, M.V., B.D. and J.P. were funded by the Centre Scientifique de Monaco (CSM) and a Grant from GEMLUC.

\section{Conflict of interest}

The authors declare that they have no conflict of interest. All co-authors have seen and agree with the contents of the manuscript and there is no financial interest to report.

\section{Publisher's note}

Springer Nature remains neutral with regard to jurisdictional claims in published maps and institutional affiliations.

Received: 4 June 2020 Revised: 4 September 2020 Accepted: 7 September 2020

Published online: 23 September 2020

\section{References}

1. Green, D. R. \& Evan, G. I. A matter of life and death. Cancer Cell 1, 19-30 (2002).

2. Hanahan, D. \& Weinberg, R. A. The hallmarks of cancer. Cell 100, 57-70 (2000).

3. Hanahan, D. \& Weinberg, R. A. Hallmarks of cancer: the next generation. Cell 144, 646-674 (2011). 
4. Kerr, J. F., Wyllie, A. H. \& Currie, A. R. Apoptosis: a basic biological phenomenon with wide-ranging implications in tissue kinetics. Br. J. Cancer 26 239-257 (1972).

5. Lowe, S. W. \& Lin, A. W. Apoptosis in cancer. Carcinogenesis 21, 485-495 (2000).

6. Brown, J. M. \& Attardi, L. D. The role of apoptosis in cancer development and treatment response. Nat. Rev. Cancer 5, 231-237 (2005).

7. Hassan, M., Watari, H., AbuAlmaaty, A., Ohba, Y. \& Sakuragi, N. Apoptosis and molecular targeting therapy in cancer. Biomed. Res. Int. 2014 150845 (2014).

8. Reed, J. C. Apoptosis-targeted therapies for cancer. Cancer Cell 3, 17-22 (2003).

9. Wong, R. S. Apoptosis in cancer: from pathogenesis to treatment. J. Exp. Clin. Cancer Res. 30, 87 (2011).

10. Dixon, S. J. et al. Ferroptosis: an iron-dependent form of nonapoptotic cell death. Cell 149, 1060-1072 (2012).

11. Hirschhorn, T. \& Stockwell, B. R. The development of the concept of ferroptosis. Free Radic. Biol. Med. 133, 130-143 (2019).

12. Eagle, $\mathrm{H}$. Nutrition needs of mammalian cells in tissue culture. Science $\mathbf{1 2 2}$, 501-514 (1955).

13. Eagle, $H$. The specific amino acid requirements of a human carcinoma cell (Stain HeLa) in tissue culture. J. Exp. Med. 102, 37-48 (1955).

14. Eagle, H. Amino acid metabolism in mammalian cell cultures. Science $\mathbf{1 3 0}$ 432-437 (1959).

15. Bannai, S., Tsukeda, H. \& Okumura, H. Effect of antioxidants on cultured human diploid fibroblasts exposed to cystine-free medium. Biochem Biophys. Res Commun. 74, 1582-1588 (1977).

16. Mason, H. S., Fowlks, W. L. \& Peterson, E. Oxygen transfer and electron transport by the phenolase complex1. J. Am. Chem. Soc. 77, 2914-2915 (1955).

17. Hayaishi, O., Katagiri, M. \& Rothberg, S. Mechanism of the pyrocatechase reaction. J. Am. Chem. Soc. 77, 5450-5451 (1955).

18. Halliwell, B. \& Gutteridge, J. M. Oxygen toxicity, oxygen radicals, transition metals and disease. Biochem J. 219, 1-14 (1984).

19. Girotti, A. W. Mechanisms of lipid peroxidation. J. Free Radic. Biol. Med. 1 87-95 (1985).

20. Kappus, H. in Oxidative Stress (ed Helmut Sies) 273-310 (Academic Press, 1985).

21. Ursini, F., Maiorino, M., Valente, M., Ferri, L. \& Gregolin, C. Purification from pig liver of a protein which protects liposomes and biomembranes from peroxidative degradation and exhibits glutathione peroxidase activity on phosphatidylcholine hydroperoxides. Biochim. Biophys. Acta 710, 197-211 (1982).

22. Seiler, A. et al. Glutathione peroxidase 4 senses and translates oxidative stress into 12/15-lipoxygenase dependent- and AlF-mediated cell death. Cell Metab. 8, 237-248 (2008).

23. Friedmann Angeli, J. P. et al. Inactivation of the ferroptosis regulator Gpx4 triggers acute renal failure in mice. Nat. Cell Biol. 16, 1180-1191 (2014).

24. Conrad, M. et al. Regulation of lipid peroxidation and ferroptosis in diverse species. Genes Dev. 32, 602-619 (2018).

25. Hassannia, B., Vandenabeele, P. \& Vanden Berghe, T. Targeting Ferroptosis to Iron Out Cancer. Cancer Cell 35, 830-849 (2019).

26. Li, J. et al. Ferroptosis: past, present and future. Cell Death Dis. 11, 88 (2020).

27. Brigelius-Flohe, R. \& Maiorino, M. Glutathione peroxidases. Biochim Biophys. Acta 1830, 3289-3303 (2013).

28. Meister, A. \& Anderson, M. E. Glutathione. Annu. Rev. Biochem. 52, 711-760 (1983)

29. Banerjee, R. \& Zou, C. G. Redox regulation and reaction mechanism of human cystathionine-beta-synthase: a PLP-dependent hemesensor protein. Arch. Biochem Biophys. 433, 144-156 (2005).

30. Pajares, M. A. \& Perez-Sala, D. Mammalian sulfur amino acid metabolism: a nexus between redox regulation, nutrition, epigenetics, and detoxification Antioxid. Redox Signal 29, 408-452 (2018).

31. Bannai, S. Transport of cystine and cysteine in mammalian cells. Biochim Biophys. Acta 779, 289-306 (1984)

32. Bassi, M. T. et al. Identification and characterisation of human $x \subset T$ that $\mathrm{CO}^{-}$ expresses, with 4F2 heavy chain, the amino acid transport activity system xc. Pflug. Arch. 442, 286-296 (2001).

33. Chung, W. J. et al. Inhibition of cystine uptake disrupts the growth of primary brain tumors. J. Neurosci. 25, 7101-7110 (2005)
34. Daher, B. et al. Genetic ablation of the cystine transporter $x C T$ in PDAC cells inhibits mTORC1, growth, survival, and tumor formation via nutrient and oxidative Stresses. Cancer Res. 79, 3877-3890 (2019).

35. Doxsee, D. W. et al. Sulfasalazine-induced cystine starvation: potential use for prostate cancer therapy. Prostate 67, 162-171 (2007).

36. Gout, P. W., Buckley, A. R., Simms, C. R. \& Bruchovsky, N. Sulfasalazine, a potent suppressor of lymphoma growth by inhibition of the $x(c)$ - cystine transporter: a new action for an old drug. Leukemia 15, 1633-1640 (2001).

37. Lo, M., Ling, V., Wang, Y. Z. \& Gout, P. W. The xc- cystine/glutamate antiporter: a mediator of pancreatic cancer growth with a role in drug resistance. $\mathrm{Br}$. J. Cancer 99, 464-472 (2008).

38. Maechler, P. \& Wollheim, C. B. Mitochondrial glutamate acts as a messenger in glucose-induced insulin exocytosis. Nature 402, 685-689 (1999).

39. Makowske, M. \& Christensen, H. N. Contrasts in transport systems for anionic amino acids in hepatocytes and a hepatoma cell line HTC. J. Biol. Chem. 257, 5663-5670 (1982).

40. Narang, V. S., Pauletti, G. M., Gout, P. W., Buckley, D. J. \& Buckley, A. R. Suppression of cystine uptake by sulfasalazine inhibits proliferation of human mammary carcinoma cells. Anticancer Res. 23, 4571-4579 (2003).

41. Lewerenz, J. et al. The cystine/glutamate antiporter system $x(c)(-)$ in health and disease: from molecular mechanisms to novel therapeutic opportunities. Antioxid. Redox Signal 18, 522-555 (2013).

42. Dolma, S., Lessnick, S. L., Hahn, W. C. \& Stockwell, B. R. Identification of genotype-selective antitumor agents using synthetic lethal chemical screening in engineered human tumor cells. Cancer Cell 3, 285-296 (2003).

43. Yang, W. S. et al. Regulation of ferroptotic cancer cell death by GPX4. Cell 156, 317-331 (2014).

44. Daher, B., Vučetić, M. \& Pouysségur, J. Cysteine depletion, a key action to challenge cancer cells to ferroptotic cell death. Front. Oncol. https://doi.org/ 10.3389/fonc.2020.00723 (2020).

45. Bersuker, $\mathrm{K}$. et al. The CoQ oxidoreductase FSP1 acts parallel to GPX4 to inhibit ferroptosis. Nature 575, 688-692 (2019).

46. Doll, S. et al. FSP1 is a glutathione-independent ferroptosis suppressor. Nature 575, 693-698 (2019)

47. Badgley, M. A. et al. Cysteine depletion induces pancreatic tumor ferroptosis in mice. Science $\mathbf{3 6 8}, 85-89$ (2020).

48. Dixon, S. J. et al. Pharmacological inhibition of cystine-glutamate exchange induces endoplasmic reticulum stress and ferroptosis. Elife 3, e02523 (2014).

49. Harris, I. S. et al. Deubiquitinases maintain protein homeostasis and survival of cancer cells upon glutathione depletion. Cell Metab. 29, 1166-1181 e1166 (2019).

50. Mauri, P. et al. Versatility of selenium catalysis in PHGPx unraveled by LC/ESIMS/MS. Biol. Chem. 384, 575-588 (2003)

51. Godeas, C. et al. Phospholipid hydroperoxide glutathione peroxidase (PHGPX) in rat testis nuclei is bound to chromatin. Biochem. Mol. Med. 59, 118-124 (1996).

52. Maiorino, M. et al. Functional interaction of phospholipid hydroperoxide glutathione peroxidase with sperm mitochondrion-associated cysteine-rich protein discloses the adjacent cysteine motif as a new substrate of the selenoperoxidase. J. Biol. Chem. 280, 38395-38402 (2005).

53. Ursini, F. et al. Dual function of the selenoprotein PHGPx during sperm maturation. Science 285, 1393-1396 (1999).

54. Banjac, A. et al. The cystine/cysteine cycle: a redox cycle regulating susceptibility versus resistance to cell death. Oncogene 27, 1618-1628 (2008).

55. Pras, E. et al. Mutations in the SLC3A1 transporter gene in cystinuria. Am. J. Hum. Genet. 56, 1297-1303 (1995).

56. Bartoccioni, P., Rius, M., Zorzano, A., Palacin, M. \& Chillaron, J. Distinct classes of trafficking rBAT mutants cause the type I cystinuria phenotype. Hum. Mol. Genet. 17, 1845-1854 (2008).

57. Arensman, M. D. et al. Cystine-glutamate antiporter $x \subset T$ deficiency suppresses tumor growth while preserving antitumor immunity. Proc. Natl Acad. Sci. USA 116, 9533-9542 (2019).

58. Eagle, $\mathrm{H}$. The sustained growth of human and animal cells in a protein-free environment. Proc. Natl Acad. Sci. USA 46, 427-432 (1960).

59. Schneider, $M$. et al. Absence of glutathione peroxidase 4 affects tumor angiogenesis through increased 12/15-lipoxygenase activity. Neoplasia 12, 254-263 (2010).

60. Lloyd, R. V. et al. p27kip1: a multifunctional cyclin-dependent kinase inhibitor with prognostic significance in human cancers. Am. J. Pathol. 154, 313-323 (1999). 
61. Wieser, R. J., Faust, D., Dietrich, C. \& Oesch, F. p16INK4 mediates contactinhibition of growth. Oncogene 18, 277-281 (1999).

62. Nelson, P. J. \& Daniel, T. O. Emerging targets: molecular mechanisms of cell contact-mediated growth control. Kidney Int. 61, S99-S105 (2002).

63. Faust, D. et al. p38alpha MAPK is required for contact inhibition. Oncogene 24, 7941-7945 (2005)

64. Cheung, T. H. \& Rando, T. A. Molecular regulation of stem cell quiescence. Nat. Rev. Mol. Cell Biol. 14, 329-340 (2013).

65. Wood, K. C. Mapping the pathways of resistance to targeted therapies Cancer Res. 75, 4247-4251 (2015).

66. Gujal, T. S. \& Kirschner, M. W. Hippo pathway mediates resistance to cytotoxic drugs. Proc. Natl Acad. Sci. USA 114, E3729-E3738 (2017).

67. Nussinov, R., Tsai, C. J. \& Jang, H. A new view of pathway-driven drug resistance in tumor proliferation. Trends Pharm. Sci. 38, 427-437 (2017).

68. Bar, J., Cohen-Noyman, E., Geiger, B. \& Oren, M. Attenuation of the p53 response to DNA damage by high cell density. Oncogene $\mathbf{2 3}, \mathbf{2 1 2 8 - 2 1 3 7}$ (2004)

69. Wenz, C. et al. Cell-cell contacts protect against t-BuOOH-induced cellular damage and ferroptosis in vitro. Arch. Toxicol. 93, 1265-1279 (2019).

70. Wu, J. et al. Publisher Correction: Intercellular interaction dictates cancer cell ferroptosis via NF2-YAP signalling. Nature 572, E20 (2019).

71. $\mathrm{Wu}$, J. et al. Intercellular interaction dictates cancer cell ferroptosis via NF2YAP signalling. Nature 572, 402-406 (2019).

72. Yang, W. H. \& Chi, J. T. Hippo pathway effectors YAP/TAZ as novel determinants of ferroptosis. Mol. Cell Oncol. 7, 1699375 (2020).

73. Yang, W. H. et al. The Hippo pathway effector TAZ regulates ferroptosis in renal cell carcinoma. Cell Rep. 28, 2501-2508 e2504 (2019).

74. Yang, W. H. et al. A TAZ-ANGPTL4-NOX2 axis regulates ferroptotic cell death and chemoresistance in epithelial ovarian cancer. Mol. Cancer Res. 18, 79-90 (2020).

75. Harvey, K. F., Zhang, X. \& Thomas, D. M. The Hippo pathway and human cancer. Nat. Rev. Cancer 13, 246-257 (2013).

76. Liu, C. Y. et al. The hippo tumor pathway promotes TAZ degradation by phosphorylating a phosphodegron and recruiting the SCF\{beta\}-TrCP E3 ligase. J. Biol. Chem. 285, 37159-37169 (2010).

77. Zhao, B., Li, L., Tumaneng, K. Wang, C. Y. \& Guan, K. L. A coordinated phosphorylation by Lats and CK1 regulates YAP stability through SCF(betaTRCP). Genes Dev. 24, 72-85 (2010).

78. Donato, E. et al. YAP and TAZ are dispensable for physiological and malignant haematopoiesis. Leukemia 32, 2037-2040 (2018).

79. Falk, M. H. et al. Apoptosis in Burkitt lymphoma cells is prevented by promotion of cysteine uptake. Int J. Cancer 75, 620-625 (1998).

80. Yang, W. S. et al. Peroxidation of polyunsaturated fatty acids by lipoxygenases drives ferroptosis. Proc. Natl Acad. Sci. USA 113, E4966-E4975 (2016).

81. Dixon, S. J. et al. Human haploid cell genetics reveals roles for lipid metabolism genes in nonapoptotic cell death. ACS Chem. Biol. 10, 1604-1609 (2015)

82. Doll, S. et al. ACSL4 dictates ferroptosis sensitivity by shaping cellular lipid composition. Nat. Chem. Biol. 13, 91-98 (2017).

83. Kagan, V. E. et al. Oxidized arachidonic and adrenic PEs navigate cells to ferroptosis. Nat. Chem. Biol. 13, 81-90 (2017).

84. Yuan, H., Li, X., Zhang, X., Kang, R. \& Tang, D. Identification of ACSL4 as a biomarker and contributor of ferroptosis. Biochem Biophys. Res. Commun. 478, 1338-1343 (2016)

85. Noto, A. et al. Stearoyl-CoA-desaturase 1 regulates lung cancer stemness via stabilization and nuclear localization of YAP/TAZ. Oncogene 36, 4671-4672 (2017).

86. Yi, M. et al. Correction to: emerging role of lipid metabolism alterations in Cancer stem cells. J. Exp. Clin. Cancer Res. 37, 155 (2018).

87. $\mathrm{Yi}, \mathrm{M}$. et al. Emerging role of lipid metabolism alterations in cancer stem cells. J. Exp. Clin. Cancer Res. 37, 118 (2018).

88. Gorin, Y. et al. Nox4 NAD(P)H oxidase mediates hypertrophy and fibronectin expression in the diabetic kidney. J. Biol. Chem. 280, 39616-39626 (2005).

89. Sedeek, M., Nasrallah, R., Touyz, R. M. \& Hebert, R. L. NADPH oxidases, reactive oxygen species, and the kidney: friend and foe. J. Am. Soc. Nephrol. 24, 1512-1518 (2013).

90. Rhim, A. D. et al. EMT and dissemination precede pancreatic tumor formation. Cell 148, 349-361 (2012).

91. Shibue, T. \& Weinberg, R. A. EMT, CSCS, and drug resistance: the mechanistic link and clinical implications. Nat. Rev. Clin. Oncol. 14, 611-629 (2017).
92. Gupta, P. B., Mani, S., Yang, J., Hartwell, K. \& Weinberg, R. A. The evolving portrait of cancer metastasis. Cold Spring Harb. Symp. Quant. Biol. 70, 291-297 (2005).

93. Viswanathan, V. S. et al. Dependency of a therapy-resistant state of cancer cells on a lipid peroxidase pathway. Nature 547, 453-457 (2017).

94. Lamouille, S., Xu, J. \& Derynck, R. Molecular mechanisms of epithelialmesenchymal transition. Nat. Rev. Mol. Cell Biol. 15, 178-196 (2014).

95. Lei, Q. Y. et al. TAZ promotes cell proliferation and epithelial-mesenchymal transition and is inhibited by the hippo pathway. Mol. Cell Biol. 28, 2426-2436 (2008).

96. Li, Z. et al. The Hippo transducer TAZ promotes epithelial to mesenchymal transition and cancer stem cell maintenance in oral cancer. Mol. Oncol. $\mathbf{9}$ 1091-1105 (2015).

97. Overholtzer, M. et al. Transforming properties of YAP, a candidate oncogene on the chromosome $11 \mathrm{q} 22$ amplicon. Proc. Natl Acad. Sci. USA 103, 12405-12410 (2006).

98. Park, J. et al. Switch-like enhancement of epithelial-mesenchymal transition by YAP through feedback regulation of WT1 and Rho-family GTPases. Nat. Commun. 10, 2797 (2019).

99. Shao, D. D. et al. KRAS and YAP1 converge to regulate EMT and tumor survival. Cell 158, 171-184 (2014).

100. Tang, Y., Feinberg, T., Keller, E. T., Li, X. Y. \& Weiss, S. J. Snail/Slug binding interactions with YAP/TAZ control skeletal stem cell self-renewal and differentiation. Nat. Cell Biol. 18, 917-929 (2016).

101. Sanchez-Martinez, R. et al. A link between lipid metabolism and epithelialmesenchymal transition provides a target for colon cancer therapy. Oncotarget 6, 38719-38736 (2015).

102. Pouyssegur, J., Dayan, F. \& Mazure, N. M. Hypoxia signalling in cancer and approaches to enforce tumour regression. Nature 441, 437-443 (2006).

103. Tam, S. Y., Wu, V. W. C. \& Law, H. K. W. Hypoxia-Induced EpithelialMesenchymal Transition in Cancers: HIF-1a and Beyond. Front. Oncol. 10, 486 (2020).

104. Miess, H. et al. The glutathione redox system is essential to prevent ferroptosis caused by impaired lipid metabolism in clear cell renal cell carcinoma. Oncogene 37, 5435-5450 (2018).

105. Zou, Y. et al. A GPX4-dependent cancer cell state underlies the clear-cell morphology and confers sensitivity to ferroptosis. Nat. Commun. 10, 1617 (2019).

106. Panzilius, E. et al. Cell density-dependent ferroptosis in breast cancer is induced by accumulation of polyunsaturated fatty acid-enriched triacylglycerides. bioRxiv, 417949. Preprint at https://doi.org/10.1101/417949 (2019).

107. Jolly, M. K. et al. Implications of the Hybrid Epithelial/Mesenchymal Phenotype in Metastasis. Front Oncol. 5, 155 (2015).

108. Nieto, M. A., Huang, R. Y., Jackson, R. A. \& Thiery, J. P. Emt: 2016. Cell 166 $21-45$ (2016).

109. Friedl, P. \& Mayor, R. Tuning collective cell migration by cell-cell junction regulation. Cold Spring Harb. Perspect. Biol. https://doi.org/10.1101/ cshperspect.a029199 (2017)

110. Artinian, N. et al. Phosphorylation of the hippo pathway component AMOTL2 by the mTORC2 kinase promotes YAP signaling, resulting in enhanced glioblastoma growth and invasiveness. J. Biol. Chem. 290 19387-19401 (2015)

111. Liang, N. et al. Regulation of YAP by mTOR and autophagy reveals a therapeutic target of tuberous sclerosis complex. J. Exp. Med. 211, 2249-2263 (2014).

112. Sciarretta, S. et al. mTORC2 regulates cardiac response to stress by inhibiting MST1. Cell Rep. 11, 125-136 (2015).

113. Gan, W. et al. LATS suppresses mTORC1 activity to directly coordinate Hippo and mTORC1 pathways in growth control. Nat. Cell Biol. 22, 246-256 (2020).

114. James, M. F. et al. NF2/merlin is a novel negative regulator of mTOR complex 1 , and activation of mTORC1 is associated with meningioma and schwannoma growth. Mol. Cell Biol. 29, 4250-4261 (2009).

115. Hansen, C. G., Ng, Y. L., Lam, W. L., Plouffe, S. W. \& Guan, K. L. The Hippo pathway effectors YAP and TAZ promote cell growth by modulating amino acid signaling to mTORC1. Cell Res. 25, 1299-1313 (2015).

116. Park, Y. Y. et al. Yes-associated protein 1 and transcriptional coactivator with PDZ-binding motif activate the mammalian target of rapamycin complex 1 pathway by regulating amino acid transporters in hepatocellular carcinoma. Hepatology 63, 159-172 (2016). 
117. Tumaneng, K. et al. YAP mediates crosstalk between the Hippo and PI(3)KTOR pathways by suppressing PTEN via miR-29. Nat. Cell Biol. 14, 1322-1329 (2012).

118. Conlon, M. et al. A Compendium of Kinetic Cell Death Modulatory Profiles Identifies Ferroptosis Regulators. bioRxiv, 826925. Preprint at https:/doi.org/ 10.1101/826925 (2019).

119. Baba, Y. et al. Protective effects of the mechanistic target of rapamycin against excess iron and ferroptosis in cardiomyocytes. Am. J. Physiol. Heart Circ. Physiol. 314, H659-H668 (2018).

120. Liu, Y. \& Deng, J. Ubiquitinationdeubiquitination in the Hippo signaling pathway (Review). Oncol. Rep. 41, 1455-1475 (2019).

121. Sousa, B., Pereira, J. \& Paredes, J. The Crosstalk Between Cell Adhesion and Cancer Metabolism. Int. J. Mol. Sci. https://doi.org/10.3390/ijms20081933 (2019)

122. Smirnova, E. et al. ATGL has a key role in lipid droplet/adiposome degradation in mammalian cells. EMBO Rep. 7, 106-113 (2006).

123. Lamming, D. W. \& Sabatini, D. M. A Central role for mTOR in lipid homeostasis. Cell Metab. 18, 465-469 (2013).

124. Hirata, E. \& Sahai, E. Tumor microenvironment and differential responses to therapy. Cold Spring Harb. Perspect. Med. https://doi.org/10.1101/cshperspect. a026781 (2017).

125. Kalluri, R. The biology and function of fibroblasts in cancer. Nat. Rev. Cancer 16, 582-598 (2016)
126. Monteran, L. \& Erez, N. The dark side of fibroblasts: cancer-associated fibroblasts as mediators of immunosuppression in the tumor microenvironment. Front Immunol. 10, 1835 (2019).

127. Liu, L. et al. Stromal myofibroblasts are associated with poor prognosis in solid cancers: a meta-analysis of published studies. PLOS ONE 11, e0159947 (2016).

128. Wang, W. et al. CD8(+) T cells regulate tumour ferroptosis during cancer immunotherapy. Nature 569, 270-274 (2019).

129. Wang, W. et al. Effector T Cells Abrogate Stroma-Mediated Chemoresistance in Ovarian Cancer. Cell 165, 1092-1105 (2016).

130. Chen, H. H. \& Kuo, M. T. Role of glutathione in the regulation of Cisplatin resistance in cancer chemotherapy. Met. Based Drugs https://doi.org/10.1155/ 2010/430939 (2010).

131. Zhang, $\mathrm{H}$. et al. CAF secreted miR-522 suppresses ferroptosis and promotes acquired chemo-resistance in gastric cancer. Mol. Cancer 19, 43 (2020).

132. Nomi, T. et al. Clinical significance and therapeutic potential of the programmed death-1 ligand/programmed death-1 pathway in human pancreatic cancer. Clin. Cancer Res. 13, 2151-2157 (2007).

133. Vogelstein, B. et al. Cancer genome landscapes. Science 339, 1546-1558 (2013).

134. Obeid, M. et al. Calreticulin exposure dictates the immunogenicity of cancer cell death. Nat. Med 13, 54-61 (2007).

135. Panaretakis, T. et al. Mechanisms of pre-apoptotic calreticulin exposure in immunogenic cell death. EMBO J. 28, 578-590 (2009). 